\title{
Modified sludge-based purification of flue gases produced by thermal power plants
}

\author{
Elvira Khusnutdinova ${ }^{1 *}$, Larisa Nikolaeva $^{1}$ \\ ${ }^{1}$ Kazan State Power Engineering University 51 Krasnoselskaya street, Kazan 420061, Russia
}

\begin{abstract}
This paper proposes an adsorption-based method of removing sulfur dioxide from the flue gases produced by the thermal power plant. A power plant waste - chemical water treatment sludge available at Kazan CHPP-1 - was used as an adsorption material. Presented here is the chemical composition of the chemical water treatment sludge for a modified sorption material to be designed therefrom. The new sorption material was trial tested for removal of sulfur dioxide from gases. This resulted in kinetic dependence and adsorption isotherm. The test results were then used to design the adsorber. The economic and environmental effect of using the chemical water treatment sludge as the sorption material was calculated.
\end{abstract}

\section{Introduction}

One of the issues that the power industry faces is the large volumes of gas emissions containing harmful gas components, namely sulfur dioxide. Major sources of sulfur dioxide emissions are thermal power plants that use solid and liquid fuels. Most of these sources release emissions from stacks at altitudes of 100-200 $\mathrm{m}$ from the ground surface resulting in their significant dispersion before entering the planetary boundary layer.

Currently, there are a significant number of methods and technologies based on various chemical and physical principles that allow cleaning the flue gases of thermal power plants from harmful components. Dry (filtration, adsorption, thermal and catalytic oxidation, electronbeam exposure), combined (adsorption-catalytic, absorption-catalytic) and wet (chemisorption, absorption, washing) cleaning methods are used [1]. The performance of these methods is quite high; however, some are characterized by high cost of equipment and operation, as well as the complexity of processes.

One of the most effective methods for cleaning gas emissions is microporous sorbents-based adsorption. This technology ensures the content of harmful substances in the off-gases at the level of standards for emission factor for boilers of a certain steam capacity and various types of fuel (GOST R 50831-95). Performance indicators of adsorption treatment of gas emissions depend on the properties of adsorbents, the choice of which is determined by the power consumption and material costs of cleaning [2].

The adsorbent shall exhibit a high sorption capacity and have a relatively low cost. Available raw materials shall be used for its production. Various materials of natural and artificial origin, such as activated carbons, alumina gels, silica gels, and zeolites, are used as adsorbents for cleaning process gaseous emissions. These materials appear to show selectivity in capturing combustion products [3]. Therefore, the production of new low-cost sorbents for cleaning gas emissions in plants and factories is a requirement of the day.

This paper proposes to use dried chemical water treatment sludge produced by Kazan CHPP-1 as a sorbent for gas emissions. The chemical water treatment sludge is a waste of many tons produced by power plants in clarifiers during the physical and chemical processes of coagulation and lime clarification. Its use in technologies for cleaning gas emissions may be one of the priorities, since the volume of carbonate sludge requiring disposal increases every year.

The chemical water treatment sludge of Kazan CHPP1 has the following chemical composition:

\begin{tabular}{|c|c|}
\hline \multicolumn{2}{|c|}{ Cations, \% (by weight): } \\
\hline $\mathrm{Fe}^{3+}$. & $\ldots \ldots .44 \pm 0.15$ \\
\hline $\mathrm{Mg}^{2+}$ & $\ldots \ldots . .11 .0 \pm 2.2$ \\
\hline $\mathrm{Cu}^{3+}$. & $\ldots .0 .05 \pm 0.014$ \\
\hline $\mathrm{Ni}^{2+}$. & $\ldots .0 .09 \pm 0.003$ \\
\hline $\mathrm{Zn}^{2+}$. & $\ldots 0.038 \pm 0.013$ \\
\hline $\mathrm{Mn}^{2+}$ & $\ldots \ldots .1 .2 \pm 0.407$ \\
\hline $\mathrm{Cr}^{3+}$. & $.0 .001 \pm 0.0003$ \\
\hline $\mathrm{Pb}^{2+}$. & $.0 .002 \pm 0.0003$ \\
\hline $\mathrm{Cd}^{2+}$. & $\ldots . . .0 .26 \pm 0.08$ \\
\hline $\mathrm{Hg}^{2+}$. & .........Traces \\
\hline
\end{tabular}

Anions, \% (by weight):

$\mathrm{CO}_{3}^{2-}$

$\mathrm{SO}_{4}^{2-}$ $81.5 \pm 10.6$

$\mathrm{OH}^{-}$ $6.5 \pm 0.85$

$\mathrm{SiO}_{3}^{2+}$

$11.4 \pm 3.61$

$\mathrm{PO}_{4}^{3-}$ $0.6 \pm 0.11$ ..None

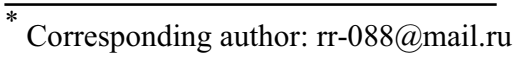


Specification of chemical water treatment sludge: bulk density $-560 \mathrm{~kg} / \mathrm{m}^{3}$; total pore volume $-0.375 \mathrm{~cm}^{3} / \mathrm{g}$; effective size fraction radius -0.05 to $1.40 \mathrm{~mm}$; sludge humidity $-3 \%$, ash content $-89 \%$ [4], organic carbon content $-11 \%$, humic substances $-12 \%$. The number of the latter was determined from the total weight of the sample, which were detected by gas chromatographymass spectrometry [5]. It is the significant specific surface of the sludge and the presence of a large number of active sites on it that determine its sorption properties.

The technologies for cleaning gas emissions produced by plants and factories use pellet adsorber beds. Therefore, to reduce the hydraulic resistance in the bed, through which gas emissions are passed, pelletized adsorbents are used. The authors propose in this paper to modify the chemical water treatment sludge into pellets. To produce pellets, fine sludge with a particle size ranged 0.01 to $0.09 \mathrm{~mm}$ is mixed with sodium silicate at a mass and volume ratio of $2: 1$, respectively. This ratio was picked experimentally [6]. At a lower ratio, the sludge is partially impregnated with sodium silicate, the adsorption capacity drops and the pellets crumble during subsequent firing. With a larger ratio, the adsorption capacity does not increase significantly and the binder is overspent. Then the mixture is brought to a homogeneous matter by rolling. The obtained pellets are soaked in a muffle furnace for 3 hours [7]. The processing temperature varies from 100 to $450^{\circ} \mathrm{C}$ in $50^{\circ} \mathrm{C}$ increments. Cooling is carried out to room temperature in a desiccator. The new modified sorption material is called pelletized sorption material (PSM). The PSM granules are 1-3.5 $\mathrm{mm}$ in size and are characterized by an abrasion resistance of $78 \%$, and average hydrophilic properties.

A dummy installation with a fixed PSM bed made at the Department of Water and Fuel Technology of the Kazan State Power Engineering University was used for studying the sorption properties of PSM in a gas atmosphere that is close in composition to the flue gases produced by TPPs (Figure 1).

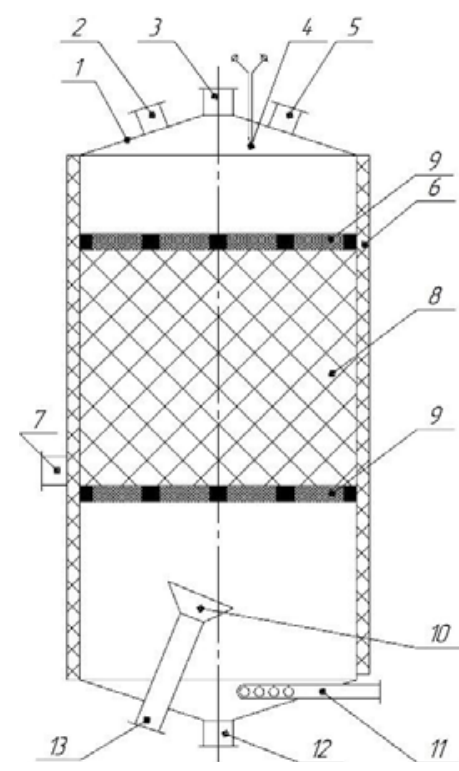

Fig. 1. The pelletized sorption material (PSM) fixed bed reaction column

1 - housing; 2 - adsorbent discharge pipe; 3 purified gas discharge pipe; 4 - thermocouple; 5 - hatch for loading adsorbent; 6 - electric heater; 7 - hatch for unloading adsorbent; 8 - PSM bed; 9 - steel mesh with holes max. $1 \mathrm{~mm}$ in diameter; 10 - sprayer, 11 - steam supply pipe; 12 - condensate discharge pipe; 13 - gas mixture supply pipe.

The reaction column is made of stainless steel to resist to corrosive environments. The gas mixture was supplied and discharged through pipes 3 and 13. The gas mixture supply pipe 13 is threaded to the sprayer 10 . This ensures a uniform distribution of gases over the PSM bed 8 on the steel mesh 9. The steel mesh 9 above the PSM bed prevents particles from carrying over. A thermostat with an electric heater 6 was used to heat up the column. The gas temperature was $50^{\circ} \mathrm{C}$, which was controlled by a chromel-drop type thermocouple.

The gas composition throughout the trial was as follows: $\mathrm{O}_{2}-4$ to $5.7 \% ; \mathrm{N}_{2}-75$ to $78 \% ; \mathrm{H}_{2} \mathrm{O}$ to $3 \%$. The remaining volume of the gas mixture was $\mathrm{CO}_{2}$. In the process of sorption, the concentration of sulfur dioxide varied in the range of $0-5,500 \mathrm{mg} / \mathrm{m}^{3}$. The gas flow rate reduced to normal conditions was $4 \times 10^{-4} \mathrm{~m}^{3} / \mathrm{s}$ [2]. Sulfur dioxide was produced in the laboratory by reaction:

$$
\mathrm{Na}_{2} \mathrm{SO}_{3}+2 \mathrm{HCl} \rightarrow 2 \mathrm{NaCl}+\mathrm{H}_{2} \mathrm{O}+\mathrm{SO}_{2} \uparrow
$$

The results of testing the PSM sorption properties toward sulfur dioxide show that the adsorption capacity of PSM effects during the first minutes of contact and after 16 minutes reaches $140 \mathrm{mg} / \mathrm{g}$ for sulfur dioxide. The adsorption capacity is $14 \mathrm{wt} \%$. Figure 2 illustrates how the sorption capacity of PSM kinetically changes with time. 


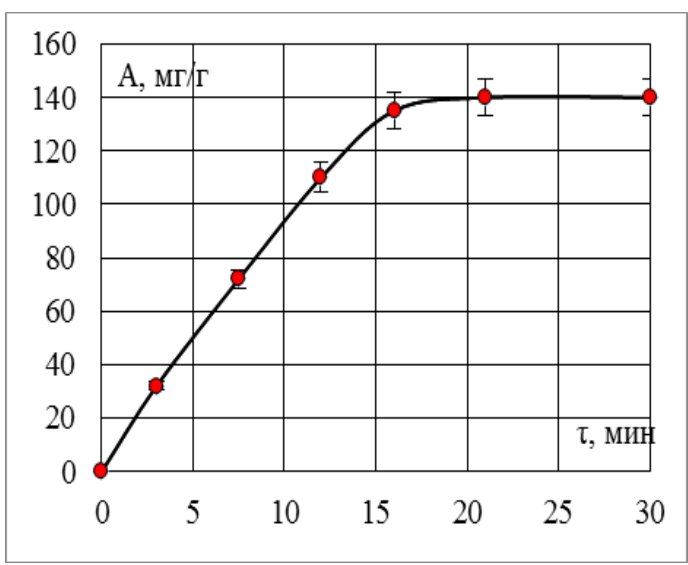

Fig. 2. The PSM sulfur dioxide adsorption kinetic curve

The optimal parameters of industrial treatment plants can be obtained using the sorption isotherm - the sorption capacity $A$ vs. the change in the $\mathrm{SO}_{2}$ concentration, as is shown in Figure 3. The adsorption isotherm corresponds to the L-type Langmuir isotherm. The convex shape of the isotherm confirms effective physical adsorption. The adsorption isotherm is best described by the Freundlich equation $\mathrm{A}=2.11 \mathrm{C}^{0.54}\left(\mathrm{R}_{2}>0.99\right)$ (Figure 4).

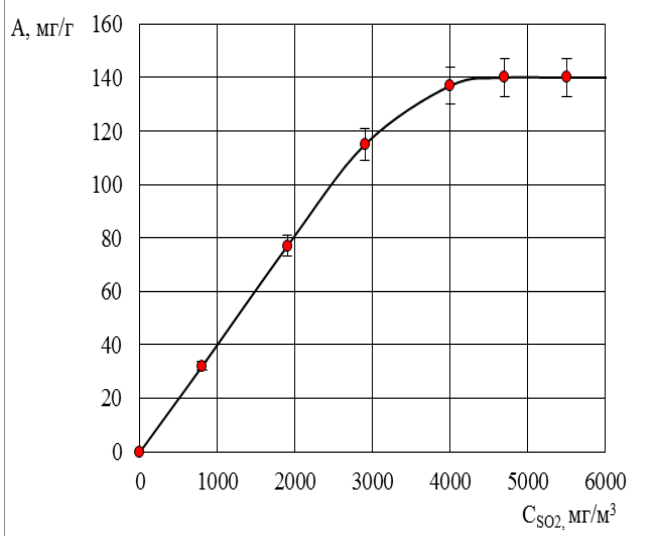

Fig. 3. The isotherm of $\mathrm{SO}_{2}$ adsorption by pelletized sorption material

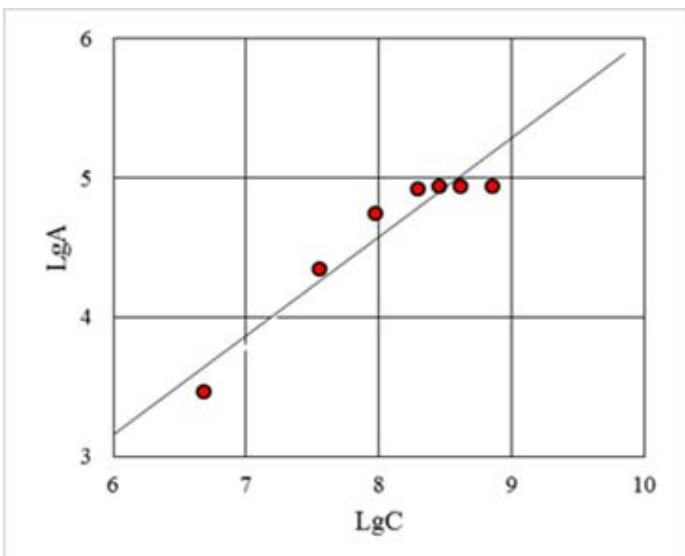

Fig. 4. The isotherm of $\mathrm{SO}_{2}$ adsorption by pelletized sorption material and its type in logarithmic coordinates
The tests resulted in the following characteristics of the modified sludge - pelletized sorption material: the $\mathrm{SO}_{2}$ adsorption capacity is $140 \mathrm{mg} / \mathrm{g}$, the total pore volume is $0.450 \mathrm{~cm}^{3} / \mathrm{g}$, the specific surface area is 720 $\mathrm{m}^{2} / \mathrm{kg}$, and the abrasion strength is $78 \%$. The efficiency of PSM-based $\mathrm{SO}_{2}$ removal was $99 \%$.

The pelletized sorption material exhibits a high porosity, which is especially important when using its adsorption properties in the gas transmission mode. PSM can be used in adsorbers in place of activated carbon with high performance.

The sulfur dioxide isotherm was then used to calculate optimal characteristics of the adsorber listed in Table 1.

Table 1. Characteristics of adsorber

\begin{tabular}{|l|c|}
\hline Parameter & Value \\
\hline Diameter, $\mathrm{m}$ & 0.4 \\
\hline $\begin{array}{l}\text { Specific surface area of PSM, } \\
\mathrm{m}^{2} / \mathrm{kg}\end{array}$ & 720.0 \\
\hline $\begin{array}{l}\text { Amount of PSM per single } \\
\text { loading, } \mathrm{kg}\end{array}$ & 47.3 \\
\hline Height of PSM bed, $\mathrm{m}$ & 2.1 \\
\hline Duration of adsorption, $\mathrm{h}$ & 82.4 \\
\hline
\end{tabular}

The continuity of purification is provided by two adsorbers, one of which is used for gas adsorption cleaning, the other - for sequential recovery of PSM bed.

Prevented environmental damage has been calculated. The amount of damage as prevented from soil and ground degradation by taking the environmental protection activities within the area under consideration for the reporting period, thousand rubles/year, is calculated by the formula:

$Y_{n p}^{n}=H_{c} S K_{\vartheta} K_{n}$

where $\boldsymbol{H}_{c}$ is the standard value of land (according to the Ministry of Environment of the Republic of Tatarstan - 206 thousand rubles/ha), $\boldsymbol{S}$ is the area of soil and land saved from degradation for the same period of time as a result of environmental actions taken, ha (according to the Kazan CHPP-1, the slurry used to produce PSM, is 0,0112 ha); $\boldsymbol{K}_{3}$ is the factor of the environmental situation and environmental importance of the area, equal to $1.9 ; \boldsymbol{K}_{\boldsymbol{n}}$ is the coefficient for specially protected areas, equal to 1 .

The calculations yield:

$Y_{n p}^{n}=206 \cdot 0,0112 \cdot 1,9 \cdot 1=4,38$ thousand rubles/year.

The amount of environmental damage prevented from soil and land degradation was 4.38 thousand rubles/year.

The low cost of PSM made from the power plant waste, i.e. chemical water treatment sludge, availability, and the possibility to be recovered, makes it possible to use for cleaning the flue gases of thermal power plants with minimal costs and maximum efficiency. The cost of PSM is 8.3 times lower than that of activated carbon. 


\section{Conclusion}

Pilot tests of modified sludge made from power plant waste for cleaning the flue gases of thermal power plants from sulfur dioxide were carried out to study kinetic regularities of the adsorption process followed by the adsorption isotherm that corresponds to the L-type Langmuir isotherm. The convex shape of the isotherm confirms effective physical adsorption.

The optimal data of a batch-operated adsorber with a fixed PSM bed were calculated: the diameter of the adsorber, the amount of PSM per charge, the height of the PSM bed, and the duration of adsorption.

Based on the test results, it was proposed to replace activated carbon in the adsorber with pelletized sorption material.

The modified gas emissions purification train with the sulfur dioxide removal bed using the carbonate sludgebased PSM was calculated for environmental and economic effect. The prevented environmental damage will amount to 4.38 thousand rubles per year. Compared with activated carbon, the cost of PSM is 8.3 times lower. The efficiency of removal of $\mathrm{SO}_{2}$ from the gas emissions using PSM was 99\%.

\section{References}

1.A. G. Laptev, M. I. Farakhov Hydro-mechanical processes in petrochemistry and power engineering. Kazan: Kazan State University Press, 2008. p. 729.

2.L. A. Nikolaeva Research of sorption processes using chemical water purifi-cation sludge for nitrogen and sulfur oxides contained in smoke gases emit-ted from a thermal power station // Thermal Engineering. 2013. Vol. 60. No. 4. pp. 244-247

3.M. G. Ziganshin, A. A Kolesnik, A.M. Ziganshin Design of dust and gas cleaning devices. Saint Petersburg: Lan, 2014.

4. L. A. Nikolaeva, A. N. Khusnutdinov Purification of gas emissions of chem-ical industry enterprises by carbonaceous cutting / Ecology \& Industry of Russia. 2018, iss. 22 No. 8. pp. $14-18$

5. L. A. Nikolaeva, A. N. Khusnutdinov A study of the absorption of nitrogen oxides from the boiler flue gases // Thermal Engineering. 2018.Vol. 65. 8 edition. pp. 575579

6.O. K. Semakina, Yu. S. Yakusheva, A. A. Shevchenko The choice of the method of pelletizing the adsorbents from waste products // Fundamental research. 2013. No. 8-3. pp. 720-725

7. M. V. Terekhova, S. M. Rusakova Investigation of adsorption patterns of anions on the surface of red sludge // Izvestiya MGTU "MAMI". 2013. No. 3, vol. 1. pp. 147151 